\title{
NIBART: A New Interval Based Algebraic Reconstruction Technique for Error Quantification of Emission Tomography Images
}

\author{
Olivier Strauss ${ }^{1}$, Abdelkabir Lahrech ${ }^{1}$, Agnès Rico ${ }^{2}$, Denis Mariano-Goulart ${ }^{3}$, \\ and Benoît Telle ${ }^{1}$ \\ 1 LIRMM UMR CNRS 5506, Université Montpellier 2, 161, rue Ada 34392 \\ Montpellier cedex 5, France \\ strauss@lirmm.fr \\ 2 LIRIS UMR CNRS 5205, Université Claude Bernard Lyon 1, 43 avenue du 11 \\ novembre 1918, 69622 Villeurbanne, France \\ 3 CHU Lapeyronie, Service Central de Médecine Nucléaire, 371, avenue du doyen \\ Gaston Giraud 34295 Montpellier cedex 5, France
}

\begin{abstract}
This article presents a new algebraic method for reconstructing emission tomography images. This approach is mostly an interval extension of the conventional SIRT algorithm. One of the main characteristic of our approach is that the reconstructed activity associated with each pixel of the reconstructed image is an interval whose length can be considered as an estimate of the impact of the random variation of the measured activity on the reconstructed image. This work aims at investigating a new methodological concept for a reliable and robust quantification of reconstructed activities in scintigraphic images.
\end{abstract}

\section{Introduction}

In the field of nuclear medicine, the need for a reliable comparison of reconstructed activities in two regions of interest often occurs when the question is to decide whether the increase in the metabolism of a tissue is high enough, compared with normal tissue in the neighborhood, to diagnose or to exclude a particular disease. Since any reconstruction method tends to highly correlate the acquisition error, a robust comparison of reconstructed activities in two (or more) regions of interest leads to the need for a general methodology for reliably predicting the noise and resolution properties in reconstructed images.

Several solutions have been proposed in the relevant literature for quantifying the noise in the reconstructed slices. They generally assume that the reconstructed image results from some optimization of a functional that takes into account both a precise geometric description of the tomographic problem, a model of the statistical properties of the measured projections and some a-priori knowledge on the resolution of image to be reconstructed [1234]. All these works are based on an empirical or a numerical inversion of the Jacobian of the functional, that is minimized by the reconstruction problem, to analytically 
deduce the statistical properties of the reconstructed image from a presupposed Poisson noise in the projections. Such an inversion is hard to compute with accuracy for the ill-posed problem of tomography, especially when large image sizes are used. This makes necessary to simplify the projection process, thus leading to results whose accuracy and reliability is badly known and sub-optimal. Even when strong simplifying hypotheses are put forward, the proposed methods are generally too computationally expensive $1|2| 3$. In [4, Budinger et al. propose an empirical method for predicting the noise but this method concerns only reconstruction algorithms that involve a single back-projection procedure (so mostly filtered back projection).

In this article, we develop an efficient and faster approach for estimating the image reconstruction error. This approach is based on considering a new projection operator whose output is interval valued. As shown in [5], the imprecision of this output interval is highly correlated with the statistical impact of the random variations of the measures. In the present work, we keep these quantification properties by extending a classical algebraic reconstruction technique. The error quantification ability of the proposed technique is highlighted through a simulated experiment.

This paper will be structured as follows: Section 2 briefly describes the Simultaneous Iterative Reconstruction Technique (SIRT). In Section 3, we recall some interval arithmetic operations. Section 4 presents the new interval valued projection operator we use. Section 5 describes the NIBART approach. Section 6 presents an illustrative experiment highlighting the ability of the NIBART approach to provide a noise quantification.

\section{Algebraic Reconstruction Techniques}

The reconstruction problem in emission tomography consists in determining the activity distribution in each pixel of the image to be reconstructed by using noisy discrete observations of its projection. Let $\mathbf{I}=\left(I_{1}, \ldots, I_{N}\right)$ be the activity vector of the $N$ pixels to be reconstructed and $\mathbf{S}=\left(S_{1}, \ldots, S_{K}\right)$ denote the measured activity associated to each of the $\mathrm{K}$ detector positions. If no noise is assumed in the acquisition process, then the relationship between $\mathbf{S}$ and $\mathbf{I}$, known as the discrete Radon transform, is defined by:

$$
S_{k}=\sum_{n=1}^{N} \rho_{k, n} I_{n}=\mathcal{P}(\mathbf{I}),
$$

where $\mathcal{P}$ is a linear projection operator based on a $K \times N$ Radon matrix. Each $\left\{\rho_{k, n}\right\}$ element of the Radon matrix can be considered as the probability of the activity in the $n^{t h}$ pixel to be detected by the $k^{t h}$ detector. Reconstructing the activity distribution $\mathbf{I}$ from the measurement distribution $\mathbf{S}$ consists in inverting the hight dimensional ill-conditioned linear equation Eq.(1). Many algebraic methods have been proposed in the relevant literature that consists in iteratively modifying a guessed activity density to bring each reconstructed projection in 
agreement with the measured projections. The iterative modification can be additive (see e.g. 6]) or multiplicative (see e.g. [78]). In this paper, we consider the SIRT method which achieves a global additive modification of the activity because its interval extension rather straightforward. Conventionally, the SIRT algorithm can be defined by the following equation [9]

$$
\forall n \in\{1, \ldots, N\}, \quad I_{n}^{i}=I_{n}^{i-1}+\lambda \sum_{k=1}^{K} \frac{\rho_{k, n}}{\sum_{j=1}^{K} \rho_{j, n}}\left(S_{k}-\sum_{m=1}^{N} \rho_{k, m} I_{m}^{i-1}\right),
$$

where $i$ is the iteration number and $\lambda \in[0,1]$ is a relaxation parameter.

For sake of simplicity, the SIRT algorithm (see Eq.(2)) can be reformulated as :

$$
\mathbf{I}^{i}=\mathbf{I}^{i-1}+\lambda \mathcal{B}^{*}\left(\mathbf{S}-\mathcal{P}\left(\mathbf{I}^{i-1}\right)\right)
$$

where $\mathcal{B}^{*}$ is the linear normalized back-projection operator based on the transposed Radon matrix. Using a SIRT-like algorithm to solve Eq.(1) can need a very large number of iterations to complete the reconstruction. Though, when used with noisy emission tomography measurements, the iterative reconstruction process leads to images with spurious features that rises owing to the illconditionnedness of the problem. Moreover, the level of these spurious features increases with the number of iterations. This last drawback can be a severe limit for using such a method since the few techniques that are available to provides a reliable termination criterion of the algorithm are rather intricate and generally not used in clinical routine [10].

\section{Interval Arithmetic}

In this section, we briefly present the interval arithmetic operations that will be used to extend the conventional SIRT to interval valued data. In the following, $[a]=[\underline{a}, \bar{a}]$ will denote a real interval whose lower (rsp. upper) value is $\underline{a}$ (rsp. $\bar{a})$. Let $[a]$ and $[b]$ be two real intervals, the extension of addition to intervals can be achieved in two ways [11]:

- the Minkowski addition:

$$
[a] \oplus[b]=[\underline{a}, \bar{a}] \oplus[\underline{b}, \bar{b}]=[\underline{a}+\underline{b}, \bar{a}+\bar{b}],
$$

- the dual Minkowski addition:

$$
[a] \boxplus[b]=[\underline{a}, \bar{a}] \boxplus[\underline{b}, \bar{b}]=[\min (\underline{a}+\bar{b}, \bar{a}+\underline{b}), \max (\underline{a}+\bar{b}, \bar{a}+\underline{b})] .
$$

The extension of subtraction is simply obtained by: $[a] \ominus[b]=[a] \oplus(-[b])$ and $[a] \boxminus[b]=[a] \boxplus(-[b]),(-[b])$ being equal to $[-\bar{b},-\underline{b}]$.

Note that only one of the two equations $[x] \ominus[b]=[a]$ and $[x] \ominus[a]=[b]$ has a solution and that this solution is $[x]=[a] \boxplus[b]$. Moreover, the following inclusion is always true: $([a] \boxplus[b]) \subseteq([a] \oplus[b])$. 
The extension of these operations to interval valued vectors is straightforward. Let $[\mathbf{A}]=[\underline{\mathbf{A}}, \overline{\mathbf{A}}]=\left(\left[a_{1}\right], \ldots,\left[a_{N}\right]\right)$ and $[\mathbf{B}]=[\underline{\mathbf{B}}, \overline{\mathbf{B}}]=\left(\left[b_{1}\right], \ldots,\left[b_{N}\right]\right)$ be two $\mathrm{N}$-dimension interval valued vectors, the arithmetic operation $\circ$ of $[\mathbf{A}]$ and $[\mathbf{B}]$ is defined by:

$$
[\mathbf{A}] \circ[\mathbf{B}]=\left(\left[a_{1}\right] \circ\left[b_{1}\right], \ldots,\left[a_{N}\right] \circ\left[b_{N}\right]\right),
$$

with $\circ \in\{\oplus, \ominus, \boxplus, \boxminus\}$.

\section{A New Projection Operator}

In a recent paper [5] a new way for modeling the projection operators commonly used in classical reconstruction algorithms has been proposed. This approach is based on an attempt to overcome the classical problem of discretizing the continuous Radon transform by directly using a proper discrete transform called the Hough transform. Applying the Hough transform formalism to emission tomography induces a non-additive confidence measure, instead of the conventional probabilistic confidence measure, to compute the relation between the activity of a region of the image and the observed values measured by the tomographic device. As an important consequence of this replacement, the output of the projection operator is interval-valued. Moreover, as shown in [5, the length of the interval valued activity is highly correlated with the level of its random variation. In the rest of the paper, the interval valued projection operator will be denoted $\overline{\mathcal{P}}:$

$$
[\mathbf{S}]=\underline{\overline{\mathcal{P}}}(\mathbf{I}),
$$

$[\mathbf{S}]$ being the interval valued projection vector and $\mathbf{I}$ the activity vector. The interval valued projection $[\mathbf{S}]$ can be thought as the convex set of all the values that would be obtained by using a coherent family of linear projector $\mathcal{P}$, coherent meaning that they are based on the same geometrical model. We will denote $\mathcal{F}(\underline{\overline{\mathcal{P}}})$ this family: $\mathcal{F}(\underline{\overline{\mathcal{P}}})=\left\{\mathcal{P} / \forall \mathbf{I} \in \mathbb{R}^{N}, \mathcal{P}(\mathbf{I}) \in \underline{\overline{\mathcal{P}}}(\mathbf{I})\right\}$. Since the operator $\overline{\mathcal{P}}$ preserves the order, this interval valued projector can be easily extended to an interval valued input: $[\mathbf{S}]=\overline{\mathcal{P}}([\mathbf{I}])=[\underline{\mathbf{S}}, \overline{\mathbf{S}}]$, with $[\mathbf{I}]=[\underline{\mathbf{I}}, \overline{\mathbf{I}}], \underline{\mathbf{S}}$ being the lower value of $\underline{\mathcal{P}}(\underline{\mathbf{I}}), \overline{\mathbf{S}}$ being the upper value of $\overline{\mathcal{P}}(\overline{\mathbf{I}})$. When considering the same geometrical modeling, an associated interval valued backprojection operator $\underline{\overline{\mathcal{B}}}$ can be also easily derived which represents the set of all the back-projection operators associated to each of the projectors of the family $\mathcal{F}(\underline{\overline{\mathcal{P}}})$.

The most relevant explanation for the noise quantifying ability of this intervalbased projection operator is the following. $\mathcal{F}(\underline{\overline{\mathcal{P}}})$ is a coherent subset of linear projector that should provide the same output when there is no random variation in the data to be projected. When the data are noisy, then the dispersion of the different output values given by using each $\mathcal{P} \in \mathcal{F}(\underline{\mathcal{P}})$ is a marker of the impact of the noise level on the projection to be performed. 


\section{A New Interval Based Algebraic Reconstruction Technique (NIBART)}

The challenge of NIBART is to provide an interval valued reconstructed activity that keeps the noise quantification ability of the interval valued projection and back projection operators presented in Section 4. Roughly speaking, NIBART can be thought as a simple interval-based extension of the SIRT method displayed by Eq.(3). In fact, according to Eq.(3), at the $i^{\text {th }}$ iteration, the right additive correction to apply to the guessed image $\mathbf{I}^{i-1}$, for making an image $\mathbf{I}^{i}$ whose projection is closer from the measured projection $\mathbf{S}$, is likely to be $\mathbf{D}^{i}=\lambda \mathcal{B}^{*}\left(\mathbf{S}-\mathcal{P}\left(\mathbf{I}^{i-1}\right)\right)$. In short, $\mathbf{D}^{i}$ is the expected value of $\left(\mathbf{I}^{i-1}-\mathbf{I}^{i}\right)$.

Now, when considering the interval-valued projector and back-projector and an interval valued previous guess $\left[\mathbf{I}^{i-1}\right]$, the interval valued vector $\left[\mathbf{D}^{i}\right]$ defined by $\left[\mathbf{D}^{i}\right]=\lambda \overline{\mathcal{B}^{*}}\left([\mathbf{S}] \ominus \underline{\overline{\mathcal{P}}}\left(\left[\mathbf{I}^{i-1}\right]\right)\right)$ is the convex subset of all the additive corrections that can be applied to one $\mathbf{I} \in\left[\mathbf{I}^{i-1}\right]$ when considering one $\mathcal{P} \in \mathcal{F}(\underline{\mathcal{P}})$ and one $\mathcal{B} \in \mathcal{F}(\underline{\overline{\mathcal{B}}})$. The NIBART challenge is now turned into finding the most specific interval $\left[\mathbf{I}^{i}\right]$ that is in agreement with the equation $\left[\mathbf{D}^{i}\right]=\left[\mathbf{I}^{i}\right] \ominus\left[\mathbf{I}^{i-1}\right]$. As mentioned in Section 3, this interval is given by:

$$
\left[\mathbf{I}^{i}\right]=\left[\mathbf{I}^{i-1}\right] \boxplus\left[\mathbf{D}^{i}\right]=\left[\mathbf{I}^{i-1}\right] \boxplus \lambda \underline{\overline{\mathcal{B}^{*}}}\left(\mathbf{S} \ominus \underline{\overline{\mathcal{P}}}\left(\left[\mathbf{I}^{i-1}\right]\right)\right) .
$$

Note that the precise vector $\mathbf{S}$ in expression (8) can be replaced by an interval valued vector $[\mathbf{S}]$ to account for a known error in the measurement (e.g. $[\mathbf{S}]$ is the $99 \%$ confidence interval of the measurement vector $\mathbf{S}$ ). Within the NIBART method, a certain convergence criterion can be detected since empirical results show that, after a few iterations, the measured interval $[\mathbf{S}]$ intersects the projected interval $\underline{\overline{\mathcal{P}}}\left(\left[\mathbf{I}^{i}\right]\right)$. This intersection expresses the fact that there is at least one value $\mathbf{S} \in[\mathbf{S}]$, one value $\mathbf{I} \in\left[\mathbf{I}^{i}\right]$ and one projector $\mathcal{P} \in \mathcal{F}(\underline{\mathcal{P}})$ such that $\mathbf{S}=\mathcal{P}(\mathbf{I})$. We call it the weak adequacy.

What has been settled in the many experiments we carried out, and that will be illustrated in the next section, is that, whatever the convergence conditions, the reconstruction error due to statistical noise in the measurements is highly correlated with the length of the reconstructed interval valued activity.

\section{Experiments}

This section aims at illustrating the ability of the NIBART method to provide a reliable quantification of the reconstruction error induced by the statistical noise in the measurements, whatever the convergence conditions. To achieve this experiment, we have simulated 100 noisy projections of a $64 \times 64 \mathrm{Zubal}$ phantom 11 displayed in Fig $1(\mathrm{a})$. These noisy projections have been obtained by corrupting a noise free projection of the phantom (total count per projection $\approx 10$ kcounts) with a Poisson process. The 100 simulated $64 \times 64$ noisy sinograms will be considered as 100 realizations of the same projection. For each

\footnotetext{
${ }^{1}$ Available at http://noodle.med.yale.edu/zubal/
} 


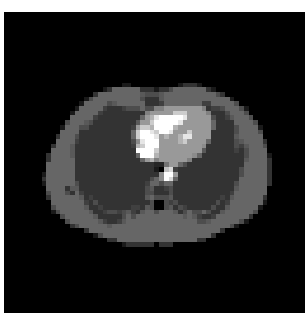

(a) Zubal phantom

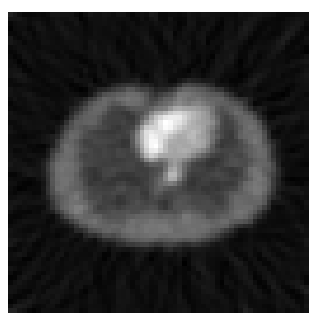

(b) 70 iterations

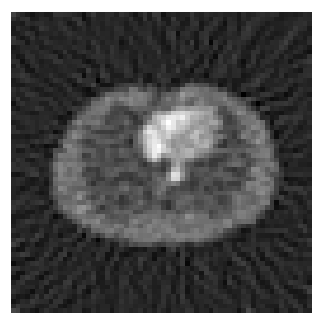

(c) 400 iterations

Fig. 1. (a) The Zubal phantom and two reconstructed median images for 70 iterations (b) and 400 iterations (c)

realization, the activity is reconstructed with the NIBART method for different number of iterations. We account for the knowledge on the noise in the projections by considering $[\mathbf{S}]$ as being the $99 \%$ confidence interval under the hypothesis of a Poisson noise. The relaxation parameter has been empirically set to $\lambda=0.024$ to ensure the convergence of the algorithm. Let $\left[\mathbf{I}^{s, t}\right]=\left[\underline{\mathbf{I}}^{s, t}, \overline{\mathbf{I}}^{s, t}\right]$ $(s \in\{1, \ldots, 100\}, t \in\{10,20, \ldots, 400\})$ be the interval valued NIBART reconstruction of the $s^{t h}$ sinogram realization with $t$ iterations. Let $\tilde{\mathbf{I}}^{s, t}=\frac{1}{2}\left(\underline{\mathbf{I}}^{s, t}+\overline{\mathbf{I}}^{s, t}\right)$ be the median of $\left[\mathbf{I}^{s, t}\right]$. Let $\Delta \mathbf{I}^{s, t}=\overline{\mathbf{I}}^{s, t}-\underline{\mathbf{I}}^{s, t}$ be the spread of $\left[\mathbf{I}^{s, t}\right]$. For each pixel $n(n \in\{1, \ldots, N\})$ we compute $\delta_{n}^{t}=\frac{1}{100} \sum_{s=1}^{100} \Delta I_{n}^{s, t}$ the mean spread of the $n^{t h}$ pixel's interval valued activity reconstructed with $t$ iterations, and $\sigma_{n}^{t}$ the standard deviation of the median values $\left(\tilde{I}_{n}^{s, t}\right)_{s \in\{1, \ldots, 100\}}$. This experiment being simulated, the convergence of the reconstructed image to the original image through the iterations can be easily depicted by computing the Euclidian distance between those two images. This distance is plotted on Fig 2(a) for one realization. For all experiments, the smallest Euclidian distance value is obtained for $t \approx 70$. We thus will consider that, after 70 iterations, the algorithm has converged. The weak adequacy occurs after always before the real convergence. In this experiment, the weak adequacy is obtained as soon as $t \geq 6$

Fig 1 presents one of the reconstructed median images for 70 and 400, that is for convergence and far from convergence. This figure shows that, since the NIBART algorithm is not regularized, the spurious features increase with the iterations.

We aim at testing wether $\delta_{n \in\{1, \ldots, N\}}^{t}$ can be considered as a measure of the noise level of the activity reconstructed activity. This is achieved by testing wether the distributions $\sigma_{n \in\{1, \ldots, N\}}^{t}$ and $\delta_{n \in\{1, \ldots, N\}}^{t}$ are correlated whatever the number $t$ of iterations. Fig $3\left(\right.$ a) plots the clouds of $\sigma_{n \in\{1, \ldots, N\}}^{70}$ versus $\delta_{n \in\{1, \ldots, N\}}^{70}$. It shows a high correlation between the statistical reconstruction error and its estimation by using the length of the reconstructed interval in convergence conditions. Fig 3(b) plots the linear (Pearson) correlation coefficient between $\sigma_{n \in\{1, \ldots, N\}}^{t}$ and $\delta_{n \in\{1, \ldots, N\}}^{t}$ for different values of $t$. Fig $3(\mathrm{~b})$ highlight the fact that, as soon as the convergence is obtained (i.e. after 70 iterations), the 


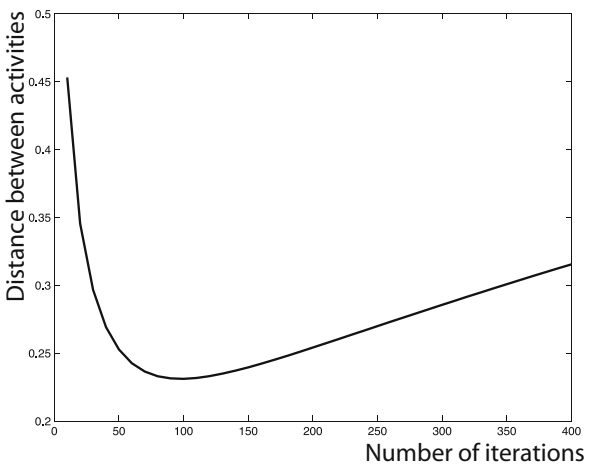

(a)

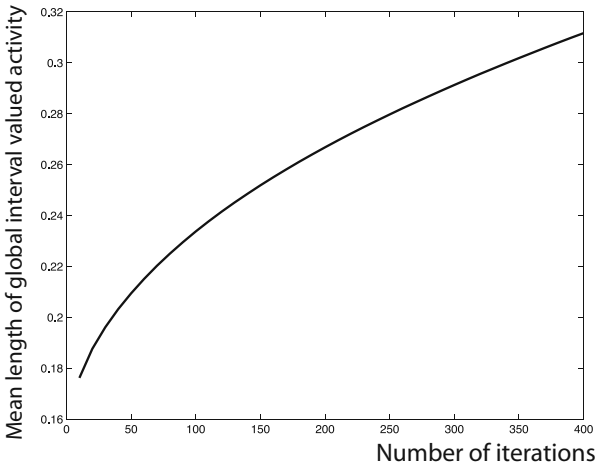

(b)

Fig. 2. (a) Distance between the simulated activity and a reconstructed median image, (b) Evolution of the mean length of the global interval valued activity through the iterations

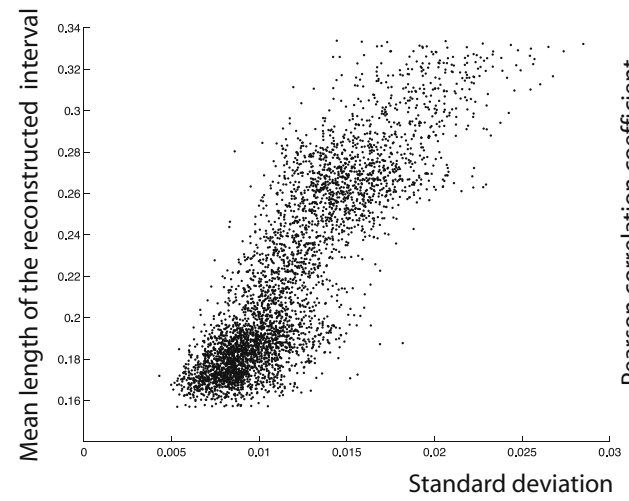

(a)

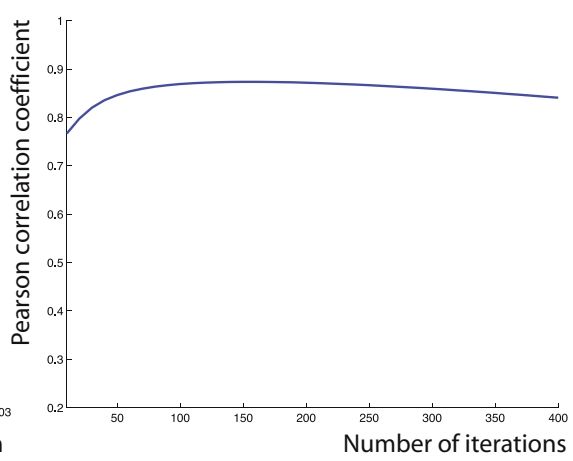

(b)

Fig. 3. (a) The standard deviation of the reconstructed activity versus its estimation by using the length of the reconstructed intervals for the $70^{\text {th }}$ iterations, (b) Evolution of the correlation coefficient through the iterations

standard deviation of the median value is highly correlated with the length of the reconstructed interval. Moreover, this correlation remains high even when the reconstructed image is far from convergence. In fact, when the convergence conditions are not fulfilled, the average length of the interval-valued reconstructed activity tends to increase (see Fig 2(b)], reflecting the fact that the influence of the noise in the measurement on the reconstructed image increases with the iterations. Note that, when the weak adequacy occurs, the correlation is already high (here $\geq 0.77$ ). 


\section{Conclusion}

In this paper, we have presented an interval based algebraic method for predicting the noise in emission tomography reconstructed images. This method is based on extending the SIRT method to account for the new modeling of the projection performed by the tomographic device presented in [5]. The main characteristic of our approach is that the reconstructed activity is interval valued. As highlighted in the simulated experiment we report here, the length of the interval associated to each pixel of the reconstructed image is highly correlated with the statistical variance of its reconstructed value. Such a noise prediction can be necessary for making reliable the comparison between the reconstructed activity in two regions of interest, this comparison being the ground of nuclear imaging diagnosis. In future work, we will consider extending this method to other iterative reconstruction techniques like EM algorithm [12. We will carry out experiments with real data obtained with a physical phantom. We will also compare our approach to the method proposed by Stayman and Fessler [2] on both simulated and real data.

\section{References}

1. Barret, H., Wilson, D., Tsui, B.: Noise properties of the EM algorithm. I: theory. Phys. Med. Bio. 39, 833-846 (1994)

2. Stayman, J., Fessler, J.: Efficient calculation of resolution and covariance for penalized-likelihood reconstruction in fully-3D SPECT. IEEE Trans. on Image Processing 23, 1543-1556 (2004)

3. Qi, J., Leahy, R.M.: A theoretical study of the contrast recovery and varaince of MAP reconstructions from PET data. IEEE Trans. Med. Imag. 18(4), 293-305 (1999)

4. Budinger, T., Derenzo, S., Greenberg, W., Gullberg, G., Huesman, R.: Quantitative potentials of dynamic emission computed tomography. J. Nucl. Med. 19, 309-315 (1978)

5. Rico, A., Strauss, O., Mariano-Goulart, D.: Choquet integrals as projection operators for quantified tomographic reconstruction. Fuzzy Sets and Systems 160, 198-211 (2009)

6. Gordon, R., Bender, R., Herman, G.: Algebraic reconstruction techniques (ART) for three dimensional electron microscopy and x-ray photography. J. Theor. Bio. 36, 105-117 (1970)

7. Dempster, A., Laird, N., Rubin, D.: Maximum likelihood from incomplete data via the EM algorithm. J. Royal Stat. Soc. 39, 1-38 (1977)

8. Hudson, H., Larkin, R.: Accelerated image reconstruction using ordered subsets of projection data. IEEE Trans. Med. Imag. 13, 601-609 (1994)

9. Lakshminarayanan, A., Lent, A.: Methods of least squares and SIRT in reconstruction. J. Theor. Bio. 76, 267-295 (1979)

10. Mariano-Goulart, D., Maréchal, P., Gratton, S., Giraud, L., Fourcade, M.: A priori selection of the regularization parameters in emission tomography by Fourier synthesis. Computerized Medical Imaging and Graphics 31, 502-509 (2007)

11. Danilov, V., Koshevoy, G.: Methods of least squares and SIRT in reconstruction. J. of Math. Anal. and App. 247, 1-14 (2000)

12. Hebert, T., Leahy, R.: A generalized EM algorithm for 3-D bayesian reconstruction from Poisson data using gibbs priors. IEEE Trans. Med. Imag. 8, 194-202 (1989) 